\title{
ASTROPHYSICAL \\ AND COSMOLOGICAL \\ CONSTRAINTS TO \\ NEUTRINO PROPERTIES
}

Edward W. Kolb, Darid N. Schramm and Michael S. Turner

The University of Chicago

and NASA/Fermilab Astrophysics Center

\begin{abstract}
The astrophysical and cosmological constraints on neutrino properties (masses, lifetimes, numbers of flavours, etc.) are reviewed.
\end{abstract}

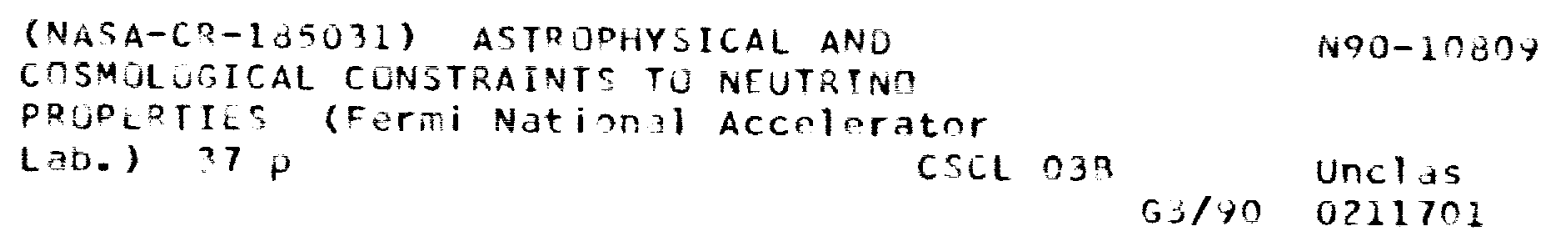

N90-10009

Prepared for Neutrino Physics, edited by Klaus Winter (Cambridge University Press, Cambridge, 1989) 
Since the 1970's with the establishment of the big bang model, it has become clear that some of the most restrictive constraints on certain neutrino properties come from astrophysical and cosmological considerations. Furthermore, in 1987 the detection of neutrinos from the supernova in the Large Magellanic Cloud provided a new "neutrino laboratory" as well as confirming our basic understanding of gravitational collapse energetics. This article will review those constraints on neutrinos derived from cosmological and astrophysical considerations.

We will first review the freeze out of neutrinos in the early Universe and derive the cosmological limits on masses for stable neutrinos. We will then use the freeze out arguments coupled with observational limits to constrain decaying neutrinos as well. We will also review the limits to neutrino properties which follow from SN198TA. We will then look at the constraint from big bang nucleosynthesis on the number of neutrino flarours. Before ending, we will briefly look at astrophysical constraints on neutrino-mixing as well as future astronomical obsertations of relerance to neutrino physics.

\section{Cosmological Mass and Decay Limits}

Cosmological limits to neutrino mass and decay properties depend on their relic number density from the early Universe. If a massive particle species remained in thermal equilibrium until the present, its abundance, $n / s \sim(m / T)^{3 / 2} \exp (-m / T)$, would be absolutely negligible because of the exponential factor ( $s=$ entropy density). If the interactions of the species freeze out (i.e., $\Gamma<H$ ) where $\Gamma$ is the interaction rate and $H$ is the cosmological expansion rate at a temperature such that $m / T$ is not much greater than 1 , the species can have a significant relic abundance today. We will now calculate that relic abundance.

First, suppose that the species is stable (or very long-lived compared to the age of the Universe when its interactions freeze out). Later we will consider the case where the species is unstable. Given that it is stable, only annihilation and inverse annihilation processes, 
e.g.,

$$
\nu \bar{\nu} \longleftrightarrow X \bar{X},
$$

can change the number of $\nu$ 's and $\bar{\nu}$ 's in a comoring volume. Here $X$ generically denotes all the species into which $\nu$ 's can annihilate. In addition, we assume that there is no asymmetry between $\nu$ 's and $\bar{\nu}$ 's.

We will also assume that all the species $X, \bar{X}$ into which $\nu, \bar{\nu}$ annihilate have thermal distributions with zero chemical potential. Because these particles will usually have additional interactions which are 'stronger' than their interactions with $p$ 's, the assumption of equilibrium for the $X^{\prime}$ 's is almost always a good one. For example, let $\bar{Y}^{-}, \overline{X^{-}}=\epsilon^{-}, \epsilon^{+}$; while the neutrinos only have weak interactions, the $\epsilon^{ \pm}$'s have weak and electromagnetic interactions.

The evolution of the number density $n_{\nu}$, can be expressed ${ }^{1}$ in terms of the total annihilation cross section $\left\langle\sigma_{A}|v|\right\rangle$

$$
\frac{d n_{\nu}}{d t}+3 H n_{\nu}=-\left\langle\sigma_{A}|v|\right\rangle\left[n_{\nu}^{2}-\left(n_{\nu}^{\mathrm{EQ}}\right)^{2}\right]
$$

This equation for the evolution of the abundance of a species is a particular form of the Riccati equation, for which there are no general, closed-form solutions. Before we solve the equation by approximate methods, let's consider the qualitative behavior of the solution. The annihilation rate $\Gamma_{A}$ varies as $n_{E Q}$ times the thermally-averaged annihilation cross section $\left\langle\sigma_{A}|v|\right\rangle$. In the relativistic regime, $\left(m_{\nu} / T \ll 3\right) n_{E Q} \sim T^{3}$, and like other rates, $\Gamma_{A}$ will vary as some power of $T$. In the non-relativistic regime, $\left(m_{\nu} / T \gg 3\right)$ $n_{E Q} \sim(m T)^{3 / 2} \exp (-m / T)$, so that $\Gamma_{A}$ decreases exponentially. In either regime, $\Gamma_{A}$ decreases as $T$ decreases, and so eventually annihilations become impotent, roughly when $\Gamma_{A} \simeq H$, which we call freeze out.

- Hot Relics: First consider the case of a particle species such that $m / T \lesssim 3$ at freeze out. In this case, freeze out occurs when the species is still relativistic and the equilibrium 
number density per comoving volume $Y_{\mathrm{EQ}} \equiv n_{\mathrm{EQ}} / s$ is not changing with time. Since $Y_{\mathrm{EQ}}$ is constant, the final value of $Y^{-}(Y \equiv n / s)$ is very insensitive to the details of freeze out, and the asymptotic value of $I^{\circ}, I(m \rightarrow \infty) \equiv Y_{\infty}$, is just the equilibrium value at freeze out:

$$
Y_{\infty}=Y_{\mathrm{EQ}}=0.278 g_{\mathrm{eff}} / g_{* S}
$$

where $g_{\text {eff }}=g$ (bosons), $0.75 g$ (fermions), and $g$ counts the internal degrees of freedom. Thus the species freezes out with order unity abundance relative to entropy $s$ (or the number density of photons). Assuming the expansion remains isentropic thereafter (constant entropy per comoving volume), the abundance of $\nu$ 's today is ( $s_{0}$ is the present entropy density)

$$
\begin{aligned}
n_{\nu} & =s_{0} \mathrm{I}_{\infty}=2970 \mathrm{Y}_{\infty} \mathrm{cm}^{-3} \\
& =825\left(g_{\mathrm{eff}} / g_{*}\right) \mathrm{cm}^{-3} .
\end{aligned}
$$

If, after freeze out, the entropy per comoving volume of the Universe should increase, say by a factor of $\gamma$, the present abundance of $\nu$ 's in a comoving volume would be diminished by $\gamma$.

A species which decouples when it is relativistic is often called a hot relic. The present relic mass density contributed by a hot relic is simple to compute:

$$
\begin{aligned}
\rho_{\nu} & =s_{0} Y_{\infty} m=2.97 \times 10^{3} Y_{\infty}(m / \mathrm{eV}) \mathrm{eV} \mathrm{cm}^{-3} \\
\Omega_{\nu} h^{2} & =7.83 \times 10^{-2}\left[g_{\text {eff }} / g_{*}\right](\mathrm{m} / \mathrm{eV})
\end{aligned}
$$

Based upon the present age of the Universe we know that $\Omega_{0} h^{2} \lesssim 1$; applying this bound to the contribution of the species $\nu$ to $\Omega_{0} h^{2}$ we obtain a cosmological bound to the mass of the $\nu$ :

$$
m \lesssim 12.8 \mathrm{eV}\left[g_{* S}\left(x_{f}\right) / g_{\mathrm{eff}}\right]
$$


Light (mass $\lesssim \mathrm{MeV}$ ) neutrinos decouple when $T \sim$ few $\mathrm{MeV}$, and $g_{*} s=g_{*}=10.75$. For a single, 2-component neutrino species $g_{\mathrm{eff}}=2 \times(3 / 4)=1.5$, so that $g_{\mathrm{eff}} / g_{*} s=0.140$. This implies that

$$
\begin{aligned}
\Omega_{\nu} h^{2} & =\frac{m_{\nu}}{91.5 \mathrm{eV}^{\top}} \\
m_{\nu} & \lesssim 91.5 \mathrm{eV} .
\end{aligned}
$$

This cosmological bound to the mass of a stable, light neutrino is often referred to as the Cowsik-McClelland bound. ${ }^{2}$ (In their original paper, Coswik and McClelland consider a 4-component neutrino $(g=4)$, and took $\Omega<3.8, h=1 / 2$ and $T_{\nu}=T$, which resulted in the bound $m \lesssim 8 \mathrm{eV}$.)

If there are more than one light $(\lesssim \mathrm{MeV})$ species, this bound applies to the sum of the masses of the light neutrinos.

- Cold Relics: Now consider the more difficult case where freeze out occurs when the species is non-relativistic $(m / T \gtrsim 3)$, and $Y_{\mathrm{EQ}}$ is decreasing exponentially with $m / T$. In this case the precise details of freeze out are important.

First we will parameterize the temperature dependence of the annihilation cross section. On general theoretical grounds we expect the annihilation cross section to have the velocity dependence $\sigma_{A}|v| \propto v^{p}$, where $p=0$ corresponds to $s$-wave annihilation, $p=2$ to $p$-wave annihilation, etc. Since $\langle v\rangle \sim T^{1 / 2},\left\langle\sigma_{A}|v|\right\rangle \propto T^{n}, n=0$ for $s$-wave annihilation, $n=1$ for $p$-wave annihilation, etc. Therefore we parameterize $\left\langle\sigma_{A}|v|\right\rangle$ as

$$
\left\langle\sigma_{A}|v|\right\rangle \equiv \sigma_{0}(T / m)^{n}
$$

With this parameterization, the Boltzmann equation for the abundance of $\nu$ 's becomes,

$$
d Y / d x=-\lambda x^{-n-2}\left(Y^{2}-Y_{\mathrm{EQ}}^{-2}\right),
$$

where

$$
x=m / T \text {. }
$$




$$
\begin{aligned}
\lambda & =0.264\left(g_{* s} / g_{*}^{1 / 2}\right) m_{P L} m \sigma_{0} \\
Y_{E Q} & =0.145\left(g / g_{* s}\right) x^{3 / 2} \epsilon^{-x} .
\end{aligned}
$$

As shown in ref. 1, eq. 12 can be solved approximately to good accuracy where it is found that

$$
Y_{\curvearrowright}=\frac{3.79(n+1) x_{f}^{n+1}}{\left(g_{\star} s / g_{*}^{1 / 2}\right) m_{P L} m \sigma_{0}} .
$$

where

$$
\begin{array}{r}
x_{f} \simeq \ln \left[0.038(n+1)\left(g / g_{\star}^{1 / 2}\right) m_{P L} m \sigma_{0}\right] \\
-(n+1 / 2) \ln \ln \left[0.038(n+1)\left(g / g_{*}^{1 / 2}\right) m_{P L} m \sigma_{0}\right]
\end{array}
$$

As with a hot relic, the present number density and mass density of cold relic $\nu$ 's is easy to compute,

$$
\begin{aligned}
n_{\nu 0} & =s_{0} Y_{\infty}=2970 I_{\infty} \mathrm{cm}^{-3} \\
& =1.13 \times 10^{4} \frac{(n+1) x_{f}^{n+1}}{\left(g_{* s} / g_{*}^{1 / 2}\right) m_{P L} m \sigma_{0}} \mathrm{~cm}^{-3} \\
\Omega_{\nu} h^{2} & =1.07 \times 10^{9} \frac{(n+1) x_{f}^{n+1} \mathrm{GeV}^{-1}}{\left(g_{*} / g_{*}^{1 / 2}\right) m_{P L} \sigma_{0}} .
\end{aligned}
$$

(where the subscript $f$ denotes the freeze out value). It is very interesting to note that the relic density of $\nu$ 's is inversely proportional to the annihilation cross section and mass of the particle

$$
Y_{\infty}=\frac{3.79(n+1)\left(g_{*}^{1 / 2} / g_{* S}\right) x_{f}}{m m_{P L}\left\langle\sigma_{A}|v|\right\rangle} .
$$

The smaller its annihilation cross section, the greater its relic abundance--the weak prevail. Moreover, the present mass density only depends upon the annihilation cross section at freeze out, which for $n=0$ ( $s$-wave annihilation) is independent of temperature (and energ: ). 
Let us now look at the specific application of this to massive neutrinos $\left(m \gg \mathrm{MeT}^{-}\right)$.

Annihilation for such a species proceeds through $Z^{0}$ exchange to final states $i \bar{i}$; where $i=\nu_{L}, e, \mu, \tau, u, d, s, \ldots\left(\nu_{L}\right.$ denotes any lighter neutrino species). The annihilation cross section depends upon whether the heavy neutrino is a Dirac or Majorana species; for $T \lesssim m \lesssim M_{Z}$, the annihilation cross section is

$$
\begin{aligned}
\left\langle\sigma_{A}|v|\right\rangle_{\text {Dirac }}= & \frac{G_{F}^{2} m^{2}}{2 \pi} \sum_{i}\left(1-z_{i}^{2}\right)^{1 / 2}\left[\left(C_{V_{i}}^{2}+C_{A_{i}}^{2}\right)\right. \\
& \left.+\frac{1}{2} z_{i}^{2}\left(C_{V_{i}}^{2}+C_{A_{i}}^{2}\right)\right] \\
\left\langle\sigma_{A}|v|\right\rangle_{\text {Majorana }}= & \frac{G_{F}^{2} m^{2}}{2 \pi} \sum_{i}\left(1-z_{i}^{2}\right)^{1 / 2}\left[\left(C_{V_{i}}^{2}+C_{A_{1}}^{2}\right) \& \beta_{i}^{2} / 3\right. \\
& \left.+C_{A_{1}}^{2} 2 z_{i}^{2}\right],
\end{aligned}
$$

where $z_{i}=m_{i} / m, \beta$ is the relative velocity, and $C_{V}$ and $C_{A}$ are given in terms of the weak isospin $j_{3}$, the electric charge $q$. and the Weinberg angle $\theta_{W}$ by $C_{A}=j_{3} \cdot C_{V}=$ $j_{3}-2 q \sin ^{2} \theta_{W}$. (We have assumed that the neutrino is less massive than $M_{Z}$.)

In the Dirac case, annihilations proceed through the s-wave and $\left\langle\sigma_{A}|v|\right\rangle$ is relocity independent:

$$
\sigma_{0} \simeq c_{2} G_{F}^{2} m^{2} / 2 \pi
$$

where $c_{2} \sim 5$. Taking $g=2$ and $g_{*} \simeq 60$, from our formulae we find

$$
\begin{aligned}
x_{f} & \simeq 15+3 \ln (\mathrm{m} / \mathrm{GeV})+\ln \left(c_{2} / 5\right) \\
Y_{\infty} & \simeq 6 \times 10^{-9}\left(\frac{m}{\mathrm{GeV}}\right)^{-3}\left[1+\frac{3 \ln (m / \mathrm{GeV})}{15}+\frac{\ln \left(c_{2} / 5\right)}{15}\right]
\end{aligned}
$$

from which we compute that

$$
\Omega_{\nu \bar{\nu}} h^{2}=3(m / \mathrm{GeV})^{-2}\left[1+\frac{3 \ln (m / \mathrm{GeV})}{15}\right],
$$

where we have included the identical relic abundance of the antineutrino species $\left(\Omega_{\nu \bar{v}}=\right.$ $\left.2 \Omega_{\nu}\right)$. Note that freeze out takes place at $T_{f} \simeq m / 15 \simeq 70 \mathrm{MeV}\left(m / \mathrm{GeT}^{\mathrm{V}}\right)$ - before the interactions of light neutrinos freeze out. This is because as neutrinos annihilate and 


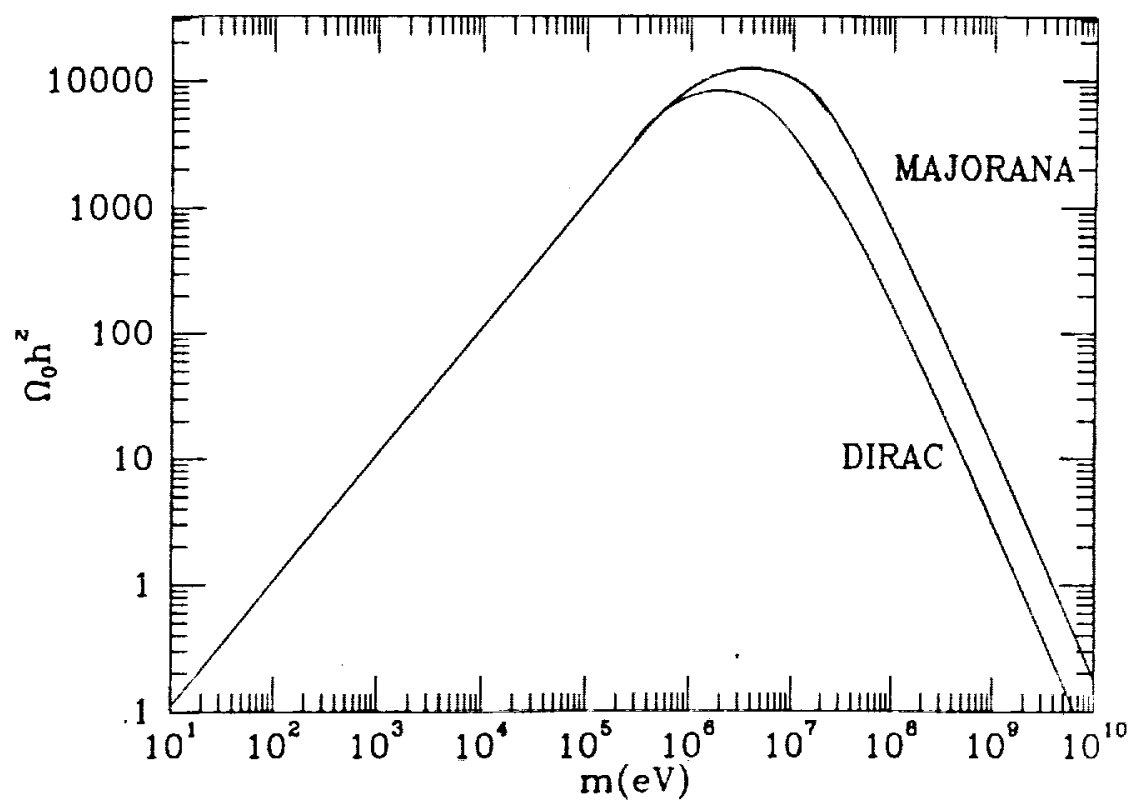

Fig. 1: The contribution to $\Omega_{0} h^{2}$ for a stable neutrino species of mass $m$ (from ref. 1).

become rare, the annihilation process quenches. Requiring $\Omega_{\nu} h^{2} \lesssim I$ we obtain the socalled Lee-Weinberg bound:

$$
m \gtrsim 2 \mathrm{GeV} \text {. }
$$

Although it is often called the Lee-Weinberg bound, the basic argument ${ }^{3}$ was noted a decade earlier by Zeldovich, Novihov and Chiu.

For the Majorana case, annihilation proceeds through both the $s$ and $p$-naves; however the formulae which obtain for $x_{f}, Y_{\infty}$ and $\Omega_{\nu} h^{2}$ are similar. In Fig. 1 we show the contribution to $\Omega_{0} h^{2}$ for a stable, massive neutrino species. For $m \lesssim \mathrm{MeV}, \Omega_{\nu} h^{2} \propto m$ as the relic abundance is constant. For $m \gtrsim \mathrm{MeV}, \Omega_{\nu} h^{2} \propto m^{-2}$ as the relic abundance decreases as $m^{-3}$. The relic mass density achieves its maximum for $m \sim \mathrm{MeV}$.

Neutrino masses less than about $92 h^{2} \mathrm{eV}$, or more than about $2 \mathrm{GeV}$ (Dirac) or about $5 \mathrm{GeV}$ (Majorana) are cosmologically acceptable.

These limits are quite impressive when compared with the laboratory limits, $\nu_{n}$ at $250 \mathrm{keV}$ and $\nu_{\tau}$ at $35 \mathrm{MeV}$, and imply that both must be below $92 \mathrm{eV}$ if they are stable. Furthermore, recent searches for the products of neut rino annihilations in the sun and earth 
by Kamiokande and Irvine-Michigan-Brookhaven (IMB) probably constrain any stable massive neutrino to be $\lesssim 12 \mathrm{GeV}$ or the high energy neutrinos produced by annihilations would have been observed. ${ }^{4}$

Before leaving stable massive neutrinos, it is worth noting that they can still be the dominant mass in the Liniverse. Relic neutrinos of a few $\mathrm{GeV}$ mass provide closure density and behave as cold dark matter. Moreover, this possibility may soon be tested by more sensitive searches for their annihilation products, and/or cryogenic detectors. Relic neutrinos of mass $\sim 30 \mathrm{eV}$ provide closure density and behave as hot dark matter. While laboratory experiments will eventually probe a $\nu_{\epsilon}$ mass as small as $10 \mathrm{eV}$, we will probably have to wait for the next nearby supernova to probe $\nu_{\mu}$ and $\nu_{\tau}$ masses in the $30 \mathrm{eV}$ range. While hot dark matter and adiabatic density perturbations (such as those produced by inflation) seem to be incompatible with observations, hot dark matter with cosmic strings (as the seed perturbations) is a very viable and interesting structure formation scenario.

\section{Unstable 1 's}

Now consider the possibility of an unstable neutrino ${ }^{5}$ species whose decay products are relativistic, even at the present epoch. It is clear that the mass density bound for such a species must be less stringent: from the epoch at which they decay (say, $z=z_{D}$ ) until the present, the mass density of the relativistic neutrino decay products decreases as $R^{-4}$, as opposed to the $R^{-3}$ had the neutrinos not decayed. Roughly speaking then, the mass density today of the decay products is a factor of $\left(1+z_{D}\right)^{-1}$ less than that of a stable neutrino species.

The precise abundance of the neutrino decay products is very easy to compute. Denote the energy density of the relativistic decay products by $\rho_{D}$, and for simplicity we will assume that they do not thermalize. The equations governing the evolution of the daughter products are:

$$
\dot{\rho}_{D}+4 H \rho_{D}=\rho_{\nu} / \tau
$$




$$
\rho_{\nu}(R)=\rho_{\nu}\left(R_{i}\right)\left(\frac{R}{R_{i}}\right)^{-3} \exp (-t / \tau)
$$

where $R_{i}, t_{i}$ is some convenient epoch prior to decay, $t_{i} \ll \tau$. The relic density of the decay products is obtained by integrating (26):

$$
\rho_{D}(t)=\rho_{\nu i} \tau^{-1}\left(\frac{R_{i}}{R}\right)^{4} \int_{t_{i}}^{t} \frac{R\left(t^{\prime}\right)}{R_{i}} \exp \left(-t^{\prime} / \tau\right) d t^{\prime} .
$$

Assuming that around the time the neutrinos decay $(t \sim \tau)$ the scale factor $R \propto t^{n}$ ( $n=1 / 2$ radiation dominated; $n=2 / 3$ matter dominated) we can evaluate this integral directly, and find that the present density of relic, relativistic particles from neutrino decays is

$$
\rho_{D}\left(t_{0}\right)=n ! \rho_{\nu}\left(t_{0}\right) \frac{R(\tau)}{R_{0}}
$$

where $\rho_{\nu}\left(t_{0}\right)$ is the present density that neutrinos and antineutrinos would have had they not decayed, and $R(\tau)$ is the value of the scale factor at the time $t=\tau$. As expected, the present energy density of the decay products is less than that of a stable neutrino species, by a factor of $n ! R(\tau) / R_{0} \sim\left(1+z_{D}\right)^{-1}$. During the matter-dominated epoch $\left(t \gtrsim 4.4 \times 10^{10}\left(\Omega_{0} h^{2}\right)^{-2} \mathrm{sec}\right), R(t) / R_{0}=2.9 \times 10^{-12}\left(\Omega_{0} h^{2}\right)^{1 / 3} t_{\mathrm{sec}}^{2 / 3}$, so that the reduction factor is

$$
n ! R(\tau) / R_{0}=2.6 \times 10^{-12}\left(\Omega_{0} h^{2}\right)^{1 / 3} \tau_{\text {sec }}^{2 / 3} .
$$

During the radiation-dominated epoch, $R(t) / R_{0}=2.4 g_{*}^{-1 / 12} \times 10^{-10} t_{\text {sec }}^{1 / 2}$, so that the reduction factor is

$$
n ! R(\tau) / R_{0}=2.1 \times 10^{-10} g_{*}^{-1 / 12} \tau_{\mathrm{sec}}^{1 / 2}
$$

Using the results of our earlier calculations for $\Omega_{\nu} h^{2}$, we obtain the following constraint 


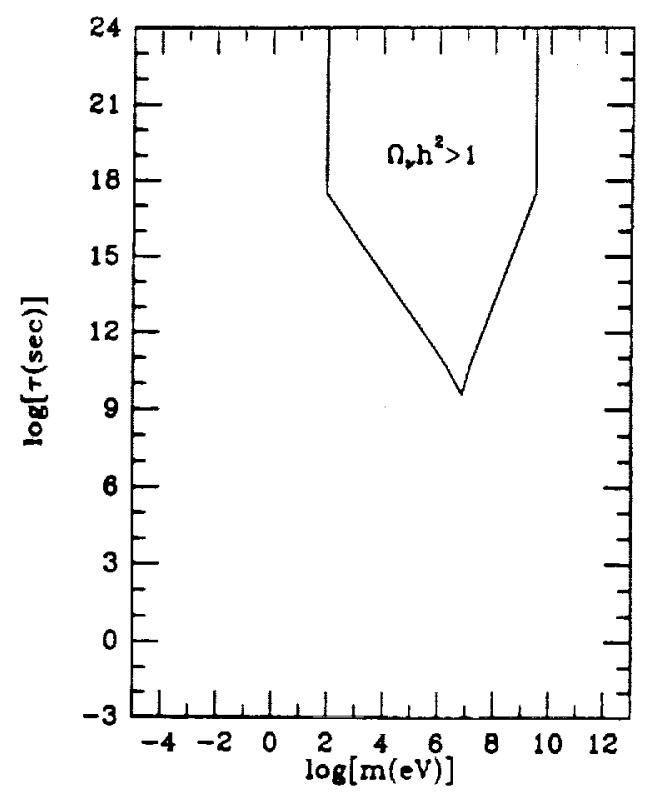

Fig. 2: The forbidden region of the neutrino mass-lifetime plane based upon the requirement that $\Omega_{\nu} h^{2} \lesssim 1$ (from ref. 1$)$.

to the epoch of decay (for neutrino masses which fall in the previously disallowed range)

$$
\begin{aligned}
& \left.m \lesssim 4 \times 10^{11} \mathrm{eVg}_{*}^{1 / 12} \tau_{\mathrm{sec}}^{-1 / 2} \quad \text { (light, } \tau \lesssim t_{\mathrm{EQ}}\right) \\
& \left.m \lesssim 4 \times 10^{13} \mathrm{eV}\left(\Omega_{0} h^{2}\right)^{-1 / 3} \tau_{\mathrm{sec}}^{-2 / 3} \quad \text { (light, } \tau \gtrsim t_{\mathrm{EQ}}\right) \\
& m \gtrsim 3 \times 10^{-5} \mathrm{GeV}_{*}^{-1 / 24} \tau_{\mathrm{sec}}^{1 / 4} \quad \text { (heary Dirac, } \tau \lesssim t_{\mathrm{EQ}} \text { ) } \\
& m \gtrsim 3 \times 10^{-6} \mathrm{GeV}\left(\Omega_{0} h^{2}\right)^{1 / 6} \tau_{\mathrm{sec}}^{1 / 3} \text { (heavy Dirac, } \tau \gtrsim t_{\mathrm{EQ}} \text { ) } \\
& m \gtrsim 7 \times 10^{-5} \mathrm{GeV}_{*}^{-1 / 24} \tau_{\mathrm{sec}}^{1 / 4} \quad \text { (heary Majorana, } \tau \lesssim t_{\mathrm{EQ}} \text { ) } \\
& m \gtrsim 8 \times 10^{-6} \mathrm{GeV}\left(\Omega_{0} h^{2}\right)^{1 / 6} \tau_{\text {sec }}^{1 / 3} \text { (heavy Majorana, } \tau \gtrsim t_{\mathrm{EQ}} \text { ). }
\end{aligned}
$$

The excluded region of the neutrino mass-lifetime plane is shown in Fig. 2. (Consideration of the formation of structure in the Universe leads to a significantly more stringent constraint to the mass density of the relativistic decay products; structure cannot grow in a radiation-dominated Universe. For a discussion of these constraints see ref. 6.)

The limits just discussed ${ }^{5}$ apply irrespective of the nature of the decay products (so long as they are relativistic). If the decay products include "visible" particles, e.g., photons, $e^{ \pm}$pairs, pions, etc., much more stringent limits can be obtained ${ }^{\top}$. We will now consider the additional constraints which apply when the decay products include a photon. (For 
the most part these same limits also apply if the decay products include $\epsilon^{ \pm}$pairs.) The limits which obtain depend both qualitatively and quantitatively upon the decay epoch, and we will consider five distinct epochs.

Before discussing these limits, it is useful to calculate the time at which the energy density of the massive neutrino species would dominate the energy density in photons. The energy density in photons is $\rho_{\gamma}=\left(\pi^{2} / 15\right) T^{4}$, and assuming the neutrinos are NR, their energy density is $\rho_{\nu}=Y_{\alpha} m s$. Taking $g_{* S} \simeq 4$, the energy densities are equal when $T \simeq 3 Y_{\propto} m$. For heavy neutrinos $Y_{\infty}$ is given by eq. 20 , and for light neutrinos. $I_{\infty} \simeq 0.04$. Thus we find that the relic neutrino energy density will exceed the photon energy density at $T / m \lesssim 0.1$ for light neutrinos, and $T / m \lesssim 2 \times 10^{-8} m_{\mathrm{GeV}}^{-3}$ for heary neutrinos. Using $t \simeq 1 \mathrm{sec} / T_{\mathrm{MeV}}^{2}$ for the age of the Universe, the epoch of matter domination (by massive neutrinos) is given by

$$
t(\mathrm{sec}) \simeq \begin{cases}10^{14}(\mathrm{~m} / 1 \mathrm{eV})^{-2} & \text { light neutrinos } \\ 3 \times 10^{9} \mathrm{~m}_{\mathrm{GeV}}^{4} & \text { heary neutrinos. }\end{cases}
$$

(Here, and throughout the following discussion, "light" will refer to neutrinos of mass less than an $\mathrm{MeV}$, and "heary" will refer to neutrinos of mass greater than an MeV, but less than $M_{Z} \cdot$ )

- $t_{U} \simeq 3 \times 10^{17} \mathrm{sec} \leq \tau:$ If the neutrino lifetime is greater than the age of the Universe, neutrinos will still be decaying at the present and decay-produced photons will contribute to the diffuse photon background. Assuming that the neutrinos are unclustered (the most conservative assumption), the differential number flux of decay-produced photons (per $\mathrm{cm}^{2}$ sr sec erg) is

$$
\frac{d \mathcal{F}_{\gamma}}{d E d \Omega}=\frac{n_{\nu} c}{4 \pi \tau H_{0}} E^{-1}\left(\frac{E}{m / 2}\right)^{3 / 2} \quad(E \leq m / 2)
$$

where for simplicity we have assumed that each decay produces one photon of energy $m / 2$ and that $\Omega_{0}=1$. Taking the number flux to be $d \mathcal{F}_{\gamma} / d \Omega \simeq E d \mathcal{F}_{\gamma} / d E d \Omega$ and $H_{0}=$ 


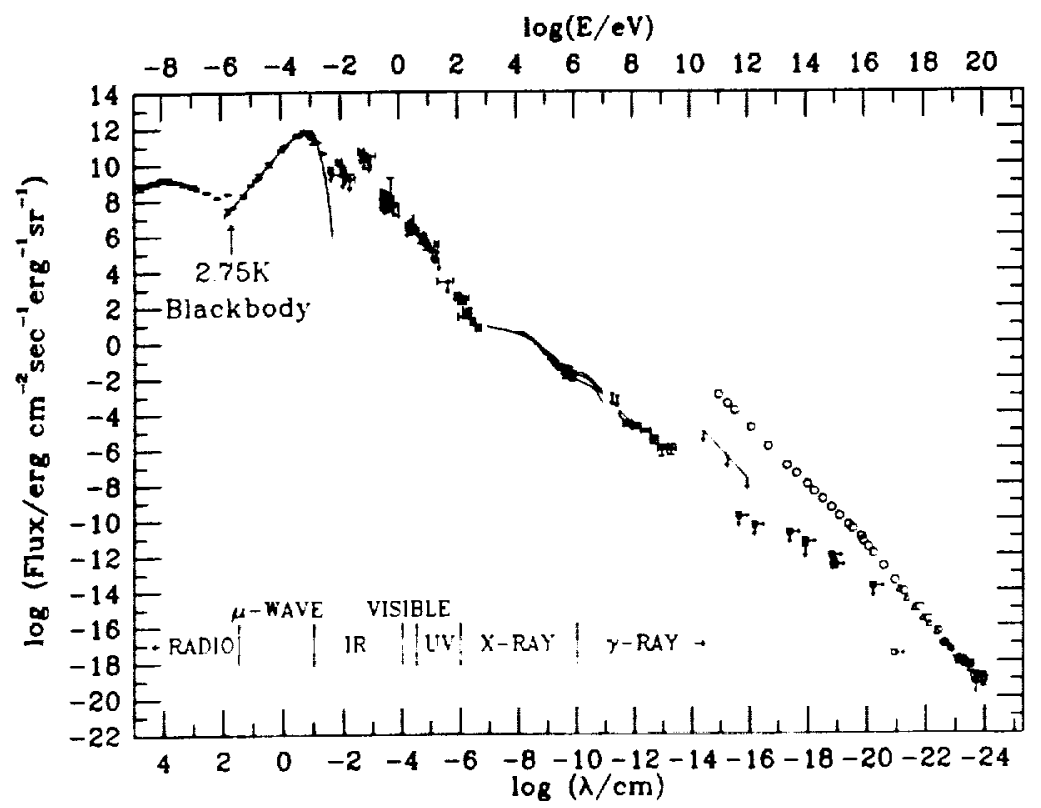

Fig. 3: The diffuse photon background. Vertical arrows indicate upper limits, and horizontal arrows indicate integrated fluxes $\left(>E\right.$.) Circles and triangles indicate the total cosmic ray flux ( ${ }^{\circ} s$, nuclei, and photons) which provides an absolute upper limit to the photon flux at the highest energies (from ref. 1).

$50 \mathrm{~km} \mathrm{sec} \mathrm{spc}^{-1}$, we find

$$
\begin{aligned}
\frac{d \mathcal{F}_{\gamma}}{d \Omega} & \simeq 10^{29} \tau_{\mathrm{sec}}^{-1} \mathrm{~cm}^{-2} \mathrm{sr}^{-1} \mathrm{sec}^{-1} \text { light neutrinos } \\
& \simeq 3 \times 10^{22} \tau_{\mathrm{sec}}^{-1} m_{\mathrm{GeV}}^{-3} \mathrm{~cm}^{-2} \mathrm{sr}^{-1} \mathrm{sec}^{-1} \text { heavy neutrinos }
\end{aligned}
$$

A summary of the observations of the diffuse photon background are shown in Fig. 3. The differential energy flux, $d \mathcal{F} / d E d \Omega$, is shown as a function of energy and wavelength. From this data, a very rough limit of

$$
\frac{d \mathcal{F}_{\gamma}}{d \Omega} \lesssim\left(\frac{\mathrm{MeV}}{E}\right) \mathrm{cm}^{-2} \mathrm{sr}^{-1} \mathrm{sec}^{-1}
$$

can be placed to the contribution of neutrino decay-produced photons to the photon background. Based upon this, the following lifetime limit results:

$$
\tau_{\mathrm{sec}} \geq \begin{cases}10^{23} \mathrm{~m}_{\mathrm{eV}} & \text { light neutrinos } \\ 10^{25} \mathrm{~m}_{\mathrm{GeV}}^{-2} & \text { heavy neutrinos, }\end{cases}
$$

applicable for neutrino lifetimes $\tau \gtrsim 3 \times 10^{17} \mathrm{sec}$. The forbidden region of the mass-lifetime plane is shown in Fig. 4. 
- $t_{\text {rec }} \simeq 6 \times 10^{12}\left(\Omega_{0} h^{2}\right)^{-1 / 2} \mathrm{sec} \leq \tau \leq t_{U}$ : If neutrinos decay after recombination, but before the present epoch, then the decay-produced photons will not interact and should appear today in the diffuse photon background. Again, for simplicity, assume that each neutrino decay produces one photon of energy $m / 2$. Then the present flux of such photons is

$$
\begin{aligned}
\frac{d \mathcal{F}_{\gamma}}{d \Omega} & =\frac{n_{\nu} c}{4 \pi} \\
& \simeq 3 \times 10^{11} \mathrm{~cm}^{-2} \mathrm{sr}^{-1} \mathrm{sec}^{-1} \quad \text { light neutrinos } \\
& \simeq 4 \times 10^{4} \mathrm{~m}_{\mathrm{GeV}}^{-3} \mathrm{~cm}^{-2} \mathrm{sr}^{-1} \mathrm{sec}^{-1} \quad \text { heary neutrinos }
\end{aligned}
$$

Where we have assumed that when the neutrino species decays, it is non-relativistic, so that each decay-produced photon today has energy $E \simeq m / 2\left(1+z_{D}\right)$, where $\left(1+z_{D}\right) \simeq$ $3.5 \times 10^{11}\left(\Omega_{0} h^{2}\right)^{-1 / 3} \tau_{\sec }^{-2 / 3}$. Comparing these flux estimates to our rough estimate of the diffuse background flux we obtain the constraints,

$$
\begin{array}{ll}
m \lesssim 2 \times 10^{6}\left(\Omega_{0} h^{2}\right)^{-1 / 3} \tau_{\mathrm{sec}}^{-2 / 3} \mathrm{eT} & \text { light neutrinos } \\
m \gtrsim S \times 10^{-3}\left(\Omega_{0} h^{2}\right)^{1 / 6} \tau_{\mathrm{sec}}^{1 / 3} \mathrm{GeV} & \text { heavy neutrinos }
\end{array}
$$

applicable for neutrino lifetimes in the range $3.5 \times 10^{11}\left(\Omega_{0} h^{2}\right)^{-1 / 3} \mathrm{sec} \lesssim \tau \lesssim 3 \times 10^{17}$ sec. For very light neutrino species the assumption that the species decays when it is non-relativistic breaks down. If the species decays after $t=t_{\text {therm }}$ and before the present epoch, and is relativistic when it decays, the decay-produced photons will be comparable in energy and in number to the CMBR photons and will cause significant distortions to the CMBR. Thus a neutrino species which decays while relativistic in the time interval $10^{6} \lesssim t \lesssim 3 \times 10^{17}$ $\mathrm{sec}$ is forbidden. The excluded region is $200 \lesssim t_{s e c} / m_{e V} \lesssim 4 \times 10^{20}\left(\Omega_{0} h^{2}\right)^{1 / 3}$, for

$$
m_{\epsilon V} \lesssim\left\{\begin{array}{l}
3.5 \times 10^{8}\left(\Omega_{0} h^{2}\right)^{-1 / 3} t_{\text {sec }}^{-2 / 3} \quad t_{s \epsilon c} \gtrsim 4.4 \times 10^{10}\left(\Omega_{0} h^{2}\right)^{-2} \\
4.6 \times 10^{6} t_{\text {sec }}^{-1 / 2} \quad t_{s \in c} \lesssim 4.4 \times 10^{10}\left(\Omega_{0} h^{2}\right)^{-2}
\end{array}\right.
$$

The forbidden region of the mass-lifetime plane is shown in Fig. 4.

- $t_{\text {therm }} \simeq 10^{6} \mathrm{sec} \leq \tau \leq t_{r \epsilon i}$ : For neutrino decays which occur during this epoch, the 


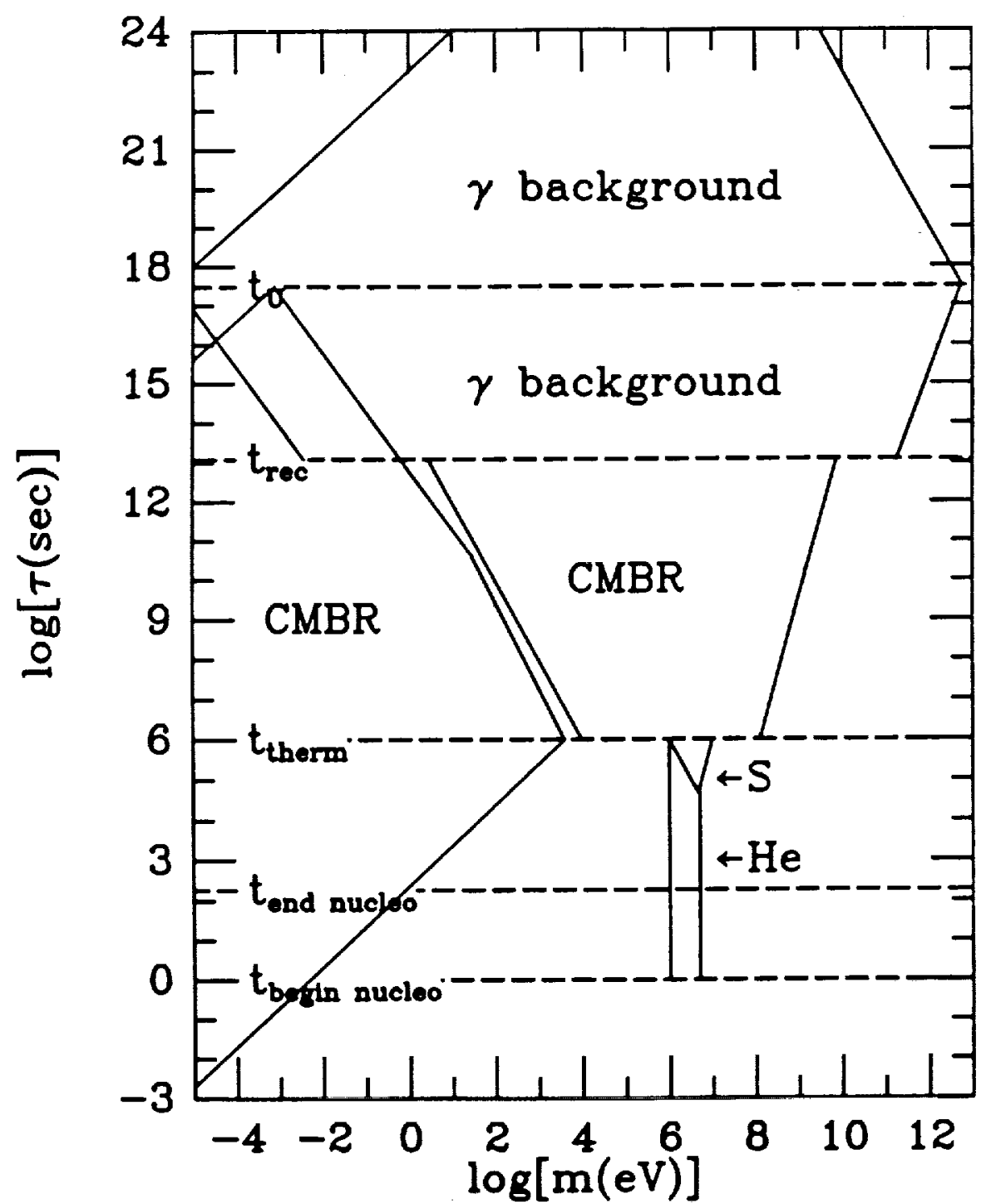

Fig. 4: Cosmological limits to the mass and lifetime of an unstable neutrino species which decays radiatively (from ref. 1). 
decay-produced photons can scatter with electrons, which can in turn scatter with Cosmic Microwave Background Radiation (CMBR) photons, thereby changing the spectral shape of the $\mathrm{CMBR}^{7}$. However, during this epoch processes which can alter the number of photons in the CMBR, e.g., the double Compton process, $\gamma+\epsilon \rightarrow \gamma+\gamma+\epsilon$, are not effective (i.e., $\Gamma<H$ ). Therefore, the result of dumping significant amounts of electromagnetic energy density from neutrino decays is a Bose-Einstein spectrum (with $\mu_{\gamma} \neq 0$ ) for the CMBR. The CMBR is to a very good precision a black body. Thus, any electromagnetic energy density resulting from neutrino decays during this epoch must be much less than that in the CMBR itself. Recalling that

$$
\begin{aligned}
\frac{\rho_{\nu}}{\rho_{\gamma}} & =\frac{m I_{\infty} s}{\rho_{\gamma}} \\
& \simeq 0.1 \mathrm{~m} / T \quad \text { light neutrinos } \\
& \simeq 2 \times 10^{-8} m_{\mathrm{GeV}}^{-3} \mathrm{~m} / T \text { hear? neutrinos, }
\end{aligned}
$$

and requiring that $\rho_{\alpha} / \rho_{\gamma} \lesssim 1$, we obtain the following limits for a neutrino species that decays during this epoch:

$$
\begin{aligned}
& m \lesssim 10^{\bar{\tau}} \tau_{\mathrm{sec}}^{-1 / 2} \mathrm{eV} \text { light neutrinos } \\
& m \gtrsim 4 \times 10^{-3} \tau_{\text {sec }}^{1 / 4} \mathrm{GeV} \text { heary neutrinos, }
\end{aligned}
$$

where we have taken $t_{\mathrm{sec}} \simeq T_{\mathrm{MeV}}^{-1 / 2}$. These limits are applicable for neutrino lifetimes in the range $10^{6} \mathrm{sec} \lesssim \tau \lesssim 10^{13} \mathrm{sec}$. The forbidden region of the mass-lifetime plane is shown in Fig. 4. (A neutrino species which decays after nucleosynthesis and produces photons of energy greater than $30 \mathrm{MeV}$ can lead to photofission of the light elements produced during nucleosynthesis; additional, more stringent bounds result ${ }^{9}$ ).

- $t_{\text {end nucleo }} \simeq 3 \min \leq \tau \leq t_{\text {therm }}$ : For neutrino decays which occur during this epoch, the decay-produced photons can be thermalized into the CMBR because both Compton and double Compton scattering are effective $(\Gamma>H)$. Howerer, in so doing the entropy per comoving volume is increased. This has the effect of decreasing the present value of $\eta$ 
relative to the standard scenario. It is known that luminous matter (necessarily baryons) provides $\Omega_{L U M} \sim 0.01$, and thus provides direct evidence that today $\eta \gtrsim 4 \times 10^{-11}$. On the other hand, primordial nucleosynthesis indicates that at the time of nucleosynthesis $\eta$ corresponded to a present value of $(3-10) \times 10^{-10}$ (ref. 8). Thus any entropy production after the epoch of nucleosynthesis must be less than a factor of $\sim 10^{-9} / 4 \times 10^{-11} \sim 30$. This leads to the limits

$$
\begin{array}{ll}
10^{9} \gtrsim m_{\mathrm{eV}} \tau_{\mathrm{sec}}^{1 / 2} & \text { light neutrinos } \\
10^{7} \gtrsim m_{\mathrm{GeV}}^{-2} \tau_{\mathrm{sec}}^{1 / 2} & \text { heavy neutrinos, }
\end{array}
$$

applicable for neutrino lifetimes in the range $200 \mathrm{sec} \lesssim \tau \lesssim 10^{6} \mathrm{sec}$. This bound too is shown in Fig. 4 (also see refs. 10).

- $t_{b e g i n \text { nucleo }} \simeq 1 \mathrm{sec} \leq \tau \leq t_{\text {end nucleo }}$. If the neutrino lifetime is longer than about a sec, then massire neutrinos can contribute significantly to the mass density of the Universe during nucleosynthesis, potentially leading to an increase in ${ }^{4} \mathrm{He}$ production. Only the equivalent of 1 additional neutrino species can be tolerated without overproducing ${ }^{4} \mathrm{He}$. One additional neutrino species is about equivalent to the energy density contributed by photons. Since the crucial epoch is when the neutron-to-proton ratio freezes out $(t \sim 1$ sec, $T \sim 1 \mathrm{MeV})$, the constraint that follows is $\left(\rho_{\nu} / \rho_{\gamma}\right)_{T \cong \mathrm{MeV}} \lesssim 1$. This results in the mass limit

$$
m \gtrsim 5 \times 10^{-3} \mathrm{GeV} \text { heavy neutrinos. }
$$

Note there is no corresponding limit for a light species because a light species is just one additional relativistic neutrino species. This limit, which is applicable to a heavy neutrino species with lifetime greater than about 1 sec is shown in Fig. 4.

- $\tau \ll 1$ sec: A neutrino species which decays earlier than about $1 \mathrm{sec}$ after the bang disappears without leaving much of a cosmological trace. Its decay products thermalize before primordial nucleosynthesis, and its only effect is to increase the entropy per comoring volume. If we understood the origin of the baryon-to-entropy ratio in great detail. and 
could predict its 'pre-nucleosynthesis' value, then we could use entropy production by the decaying neutrino species to obtain constraints for very short lifetimes.

- Astrophysical implications: Neutrino decay into visible modes can have "astrophysical" effects too (refs. 11). As the detection of neutrinos from SN 1987A dramatically demonstrated, type II supernovae are a copious source of neutrinos. The integrated flux of neutrino-decay-produced photons from type II supernovae that have occurred throughout the history of the Universe can be used to obtain a very stringent bound to acceptable neutrino masses and lifetimes.

Each type II supernova releases about $3 \times 10^{53}$ ergs of energy in thermal neutrinos with average energy about $12 \mathrm{MeV}$-or about $N_{\nu \bar{\nu}} \simeq 5 \times 10^{57}$ neutrinos and antineutrinos of each species. The historical (last $1000 \mathrm{yrs}$ ) type II rate in our own galaxy is about 1 per $30 \mathrm{yrs}$ (give or take a factor of 3 ), and the obscrvecl extragalactic rate is roughly $1.1 h^{2}$ per 100 yrs per $10^{10} L_{B \text { o. }}$. Using the mensured mean blue luminosity density of the Universe, $L_{B \odot} \sim 2.4 \mathrm{~h} \times 10^{8} L_{B \vartheta} \mathrm{Mpc}^{-3}$, this translates into a present type II rate (per volume) of $\Gamma_{S N} \simeq 2.5 h^{3} \times 10^{-85} \mathrm{~cm}^{-3} \mathrm{sec}^{-1}$. Assuming that the țpe II rate has been constant over the history of the Universe (a bold assumption), the differential photon number flux is

$$
\frac{d \mathcal{F}_{\gamma}}{d \Omega d E}=\frac{9}{5 \sqrt{2}} \frac{\Gamma_{S N} t_{U}^{2} N_{\nu \grave{\nu}}}{4 \pi\left\langle E_{\nu}\right\rangle \tau / m} \frac{1}{\left\langle E_{\nu}\right\rangle^{1 / 2} E^{1 / 2}}
$$

where for simplicity we have assumed that the supernovae neutrinos are mono-energetic, with $E_{\nu}=\left\langle E_{\nu}\right\rangle \simeq 12 \mathrm{MeV}$, that each decay-produced photon carries half the energy of the parent neutrino, and a flat Universe. For this energy spectrum $\left\langle E_{\gamma}\right\rangle=\left\langle E_{\nu}\right\rangle / 6 \simeq 2 \mathrm{MeV}$. Comparing the expected photon number flux at $\left\langle E_{\gamma}\right\rangle$,

$$
\left\langle E_{\gamma}\right\rangle \frac{d \mathcal{F}_{\gamma}}{d \Omega d E} \simeq \frac{1}{2} \frac{\Gamma_{S N} t_{U^{\prime}}^{2} N_{\nu \bar{\nu}} m}{4 \pi\left\langle E_{\nu}\right\rangle \tau},
$$

with the measured diffuse $\gamma$-ray flux at a few $\mathrm{MeV}, 3 \times 10^{-3} \mathrm{~cm}^{-2} \mathrm{sr}^{-1} \mathrm{~s}^{-1}$, we obtain the following constraint:

$$
\tau_{\mathrm{sec}} \gtrsim 5 \times 10^{12}\left(\Gamma_{S N} / 3 \times 10^{-85} \mathrm{~cm}^{-3} \mathrm{sec}^{-1}\right) m_{\mathrm{eV}} .
$$


Of course, this bound only applies to neutrino species light enough to have been produced in supernovae ( $m \lesssim 10 \mathrm{MeV}$ ) and which decay outside the envelope of the exploding star $\left(\tau_{\mathrm{sec}} \gtrsim 10^{-5} m_{\mathrm{eV}}\right)$ by the present epoch $\left(t \lesssim 10^{11} m_{\epsilon V} \mathrm{sec}\right)$. (Based upon $\gamma$-ray observations of SN 1987A made by the SMM spacecraft a similar, slightly more restrictive bound obtains. Furthermore, the lack of observed ionization around SN 19S7A by $\nu \rightarrow \nu_{\epsilon}{ }^{+} \epsilon^{-}$limits this mode for $m>1 M \epsilon V$ (see refs. 11).) This constraint is shown in Fig. 5.

For a neutrino species which decays within the envelope of the exploding star, and thereby deposits energy in the envelope a different bound can be derived. Any energy deposited by neutrino decays in the envelope will be thermalized and radiated in the visible part of the spectrum. The energy radiated by SN $1987 \mathrm{~A}$ in the visible was only about $10^{47}$ ergs, while each neutrino species carries off about $10^{53}$ ergs! The energy which is deposited in the envelope by a hypothetical, unstable neutrino species is

$$
\begin{aligned}
E_{D E P} & \simeq N_{\nu \bar{\nu}}\left\langle E_{\nu}\right\rangle \min \left[1, R_{B S G} / \tau_{L A B}\right] \\
& \simeq \min \left[10^{53} \mathrm{ergs}, 10^{48} \mathrm{~m}_{\mathrm{eV}} / \tau_{\mathrm{sec}} \mathrm{ergs}\right]
\end{aligned}
$$

where $R_{B S G} \sim 3 \times 10^{12} \mathrm{~cm}$ is the radius of the envelope of the progenitor blue super giant (Sanduleak -69 202, by name), and $\tau_{L A B}=\left\langle E_{\nu}\right\rangle \tau / m$ is the neutrino lifetime in the rest frame of the supernova. Comparing this to the observed energy of $10^{4 \pi}$ ergs, we obtain the bound

$$
\begin{aligned}
m_{\mathrm{eV}} / \tau_{\mathrm{sec}} \lesssim 0.1 \quad\left(t_{\mathrm{sec}} \gtrsim 10^{-5} m_{\mathrm{eV}}\right) \\
m_{\mathrm{eV}} \gtrsim 10^{7} \quad\left(t_{\mathrm{sec}} \lesssim 10^{-5} m_{\mathrm{eV}}\right)
\end{aligned}
$$

This constraint too is shown in Fig. 5.

A neutrino species which can decay radiatively, $\nu_{j} \longrightarrow \nu_{i}+\gamma$, necessarily has an electromagnetic coupling that may be quantified as a transition magnetic moment, $\mu_{i j}=$ $\kappa_{i j}\left(\epsilon / 2 m_{\varepsilon}\right)$. The transition magnetic moment and neutrino mass and lifetime are related 


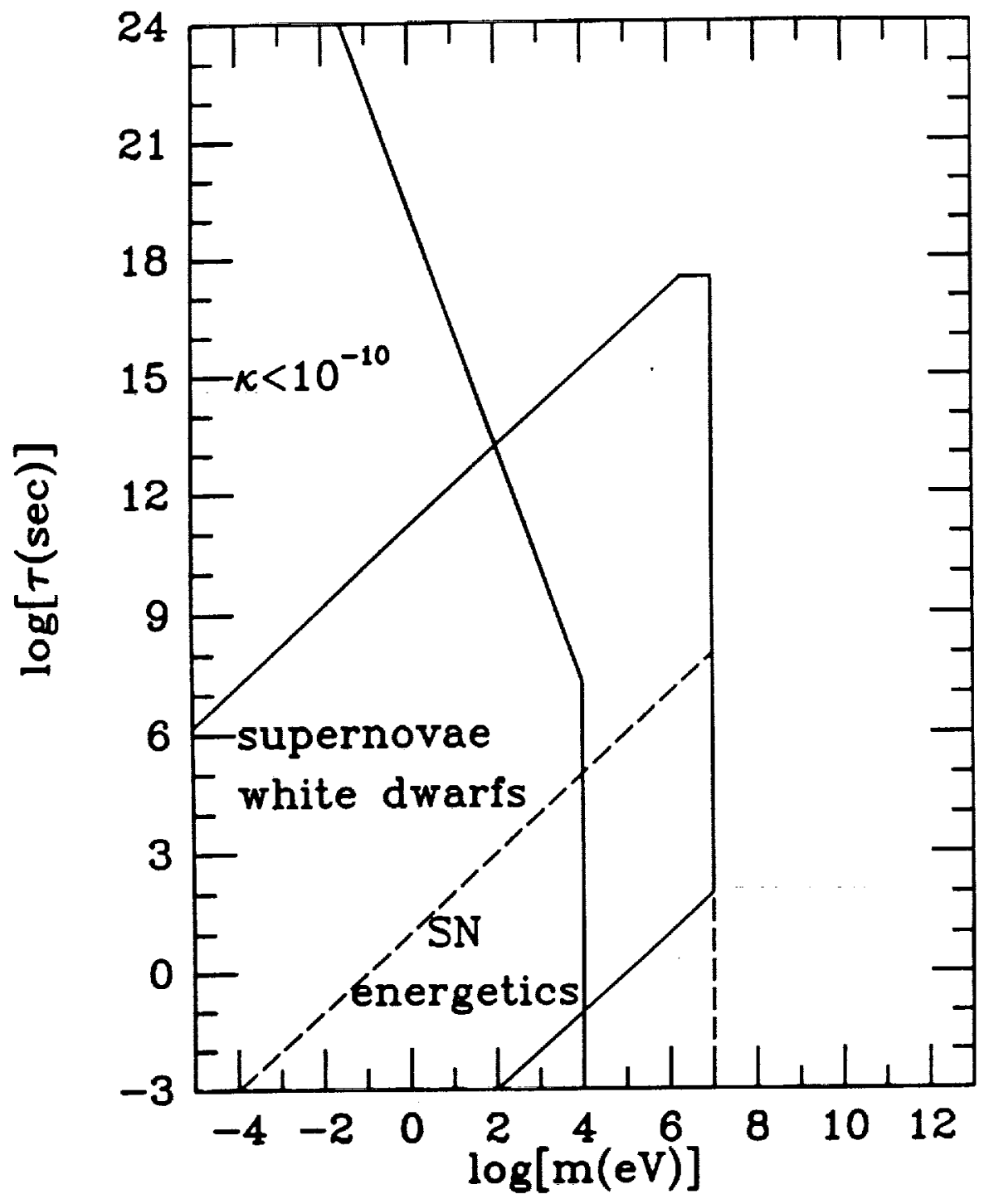

Fig. 5: Astrophysical limits to the mass and lifetime of an unstable neutrino which decays radiatively (from ref. 1). 
by

$$
\begin{aligned}
\tau^{-1} & =\alpha_{E M} \kappa^{2} m^{3} / 8 m_{\epsilon}^{2}, \\
\kappa & =0.44 \tau_{\mathrm{sec}}^{-1 / 2} m_{\mathrm{eV}}^{-3 / 2},
\end{aligned}
$$

where we have assumed $m_{j} \gg m_{i}$. The transition moment leads to an electromagnetic correction to $\nu-\epsilon$ scattering. Laboratory limits to $\nu-\epsilon$ scattering through the transition moment leads to the bound $\kappa_{\epsilon \mu} \lesssim 10^{-8}$, or

$$
\tau_{\mathrm{sec}} \gtrsim 2 \times 10^{15} m_{\mathrm{eV}}^{-3} \quad\left(\nu_{\mu} \rightarrow \nu_{\epsilon}+\gamma\right)
$$

Further, such a transition moment leads to neutrino pair emission from white dwarfs and red giants through the process plasmon $\rightarrow \nu_{i} \nu_{j}$. For $\kappa \sim 10^{-10}-10^{-11}$ plasmon $\nu \bar{\nu}$ emission can be a very significant cooling mechanism for these objects, and can effect their evolution. Based upon this, a limit of $\kappa_{i j} \lesssim 10^{-10}$ or so has been derived for neutrinos less mass than $10 \mathrm{keV}$ (see, e.g., the paper of Beg, et al. ${ }^{11}$ ). This translates to the limit

$$
\tau_{\text {sec }} \gtrsim 2 \times 10^{19} \mathrm{mel}_{\mathrm{el}}^{-3} \quad(m \lesssim 10 \mathrm{keV})
$$

All of the astrophysical and cosmological constraints just discussed are summarized in Figs. 4 and 5. These constraints serve to illustrate how a large variety of cosmological and astrophysical observations can be used to probe particle properties in regimes beyond the reach of the terrestrial laboratory.

\section{Limits To the Number of Families}

Another area where cosmological and astrophysics constraints have been important is in limiting the number of neutrino families, $N_{\nu}$. These arguments take on added importance now that accelerator experiments are beginning to check them ${ }^{12}$. The most important bound comes from big bang nucleosynthesis ${ }^{13}$. A second, very different but less stringent 
bound, comes from SN1987 $\mathrm{A}^{14}$. Let us first look at the big bang nucleosynthesis argument.

The power of big bang nucleosynthesis comes from the fact that essentially all of the input physics is well determined. The relevant temperatures, 0.1 to $0.05 \mathrm{MeV}$, are well explored in nuclear physics labs. Thus, what nuclei do under such conditions is not a matter of guesswork but is precisely known. In fact, the nuclear physics is known far better for these temperatures than it is known in the centers of stars like our sun. The temperature at the center of the sun is only a little over $1 \mathrm{keV}$. This energy is below the energy where nuclear reaction rates yield significant results in laboratory experiments, and only the long times and higher densities available in stars enable anything to take place at all! Unfortunately, for stellar astrophysics this means that nuclear reaction rates must be extrapolated to many orders of magnitude below their laboratory-measured values. The big bang laboratory does not have this problem. The reactions occur at temperatures and densities where cross sections and the like are known and well studied in the laboratory.

To calculate what happens, all one has to do is follow a gas of baryons with density $\rho_{b}$ as the Universe expands and cools. As far as nuclear reactions are concerned, the important epoch begins a little above $1 \mathrm{MeV}$ and ends a little below $100 \mathrm{KeV}$. At higher temperatures, no complex nuclei other than single neutrons and protons can exist, and the ratio of neutrons to protons, $n / p$, is just determined by thermodynamic equilibrium, $n / p=e^{-Q / T}$, where $Q=1.3 \mathrm{MeV}$ is neutron-proton mass difference. Equilibrium applies because the weak interaction rates are much faster than the expansion of the Universe at temperatures much above $i \mathrm{MeV}$. At temperatures much below $0.1 \mathrm{MeV}$, the electrostatic repulsion of nuclei prevents nuclear reactions from proceeding as fast as the cosmological expansion separates the particles.

After the weak interaction drops out of equilibrium, around $1 \mathrm{MeV}$, the ratio of neutrons to protons changes more slowly, by free neutrons decaying to protons and similar transformations of neutrons to protons via interactions with the ambient leptons. $\mathrm{By}$ the 
time the Universe reaches $0.1 \mathrm{MeV}$, the ratio is slightly below $1 / \tau$. For temperatures above $0.1 \mathrm{MeV}$, the high entropy of the Universe suppresses the abundance of nuclei. Once the temperature drops to about $0.1 \mathrm{MeV}$, nuclei begin to be present in significant amounts, starting with ${ }^{2} \mathrm{D}$ adding neutrons and protons, making ${ }^{3} \mathrm{H}$ and ${ }^{3} \mathrm{He}$. These, in turn, capture neutrons and protons to produce ${ }^{4} \mathrm{He}$ or ${ }^{3} \mathrm{H}$ and ${ }^{3} \mathrm{He}$ can collide to also yield ${ }^{4} \mathrm{He}$. Since ${ }^{4} \mathrm{He}$ is the most tightly bound nucleus (in this region of the periodic table), the flow of reactions converts almost all the neutrons that exist at $0.1 \mathrm{MeV}$ into ${ }^{4} \mathrm{He}$ (for neutron/proton ratios less than unity). The two-body chain essentially ceases there, because there are no stable nuclei at either mass-5 or mass-S. Since the baryon density at big bang nucleosinthesis is relatively low (much less than $1 \mathrm{~g} / \mathrm{cm}^{3}$ ) only reactions involving two-particle collisions occur. It can be seen that combining the most abundant nuclei neutrons, protons, and ${ }^{4} \mathrm{He}$ via 2-body interactions always lead to unstable mass-5. Even when one combines ${ }^{4} \mathrm{He}$ with rarer nuclei like ${ }^{3} \mathrm{H}$ or ${ }^{3} \mathrm{He}$, we still only get to mass- $\bar{t}$, which when hit by a proton, the most abundant nucleus around, yields mass-8. Eventually, ${ }^{3} \mathrm{H}$ radioactively decays to ${ }^{3} \mathrm{He}$, and any mass- 7 made, radioactively decays to ${ }^{\top} \mathrm{Li}$. Thus, big bang nucleosynthesis makes ${ }^{4} \mathrm{He}$ with traces of ${ }^{2} \mathrm{D},{ }^{3} \mathrm{He}$, and ${ }^{7} \mathrm{Li}$. (Also, all the protons left over that did not capture neutrons remain as hydrogen.) All other chemical elements are made later in stars and in related processes. (Stars jump the mass- 5 and -8 instability by having gravity compress the matter to sufficient densities that 3-body collisions can occur and jump the mass-5 and -8 gaps.) A neutron/proton ration of $\sim 1 / \bar{\tau}$ yields a resultant ${ }^{4} \mathrm{He}$ primordial mass fraction, $Y=2(n / p) /(n / p+1) \approx 0.25$.

The only cosmological parameter in such calculations is the density of the baryon gas at a given temperature. From the thermodynamics of the expanding Universe we know that $\rho_{b} \propto T^{3}$, thus we can relate the baryon density at $10^{11} \mathrm{~K}$ to the baryon density todar, when the temperature is about $2.75 \mathrm{~K}$. The problem is, we don't know $\rho_{b}$ today, so the calculation must be carried out for a range in $\rho_{b}$. The cosmological expansion rate depends on the total mass-energy density. For cosmological temperatures much above $1 \mathrm{el}$ the energy 
density of radiation exceeds the mass-energy density of the baryon gas. Thus, during big bang nucleosynthesis, we need the radiation density as well as the baryon density: The baryon density determines the density of the nuclei and thus their interaction rates, and the radiation density controls the expansion rate of the Universe at those times. The density of radiation is just proportional to the number of "types" of radiation. Thus, the density of radiation is not a free parameter provided we know how many types of relativistic particles exist at temperatures $\sim 0.1-1.0 \mathrm{MeV}$.

Assuming that the relativistic particles at $1 \mathrm{MeV}$ are photons, $\epsilon . \mu$, and $\tau$ neutrinos (and their antiparticles) and electrons (and positrons), the big bang nucleosynthetic yields have been calculated for a range in present $\rho_{b}$ (more precisely the baryon to photon ratio), going from less than that observed in galaxies to greater than that allowed by the observed large-scale dynamics of the Universe. The ${ }^{4} \mathrm{He}$ yield is almost independent of the baryon density, with a very slight rise in the density due to the decreasing entropy per baryon, which enables nucleosynthesis to start slightly earlier, when the neutron/proton ratio was higher. No matter what assumptions one makes about the baryon density, it is clear that ${ }^{4} \mathrm{He}$ is predicted by big bang nucleosynthesis to have to be around $25 \%$ of the mass of the Universe. This was first noted by Hoyle and Tayler ${ }^{15}$ and later found by Peebles ${ }^{16}$ and by Wagoner, Fowler, and Hoyle ${ }^{17}$. The current results do not differ in any qualitative way from Wagoner, Fowler, and Hoyle's original detailed calculations.

The fact that the observed helium abundance in all objects is about 20 to $30 \%$ is certainly a nice confirmation of these ideas. Since stars produce only a yield of $2 \%$ in all the heavy elements combined, stars cannot easily duplicate such a large ${ }^{4} \mathrm{He}$ yield. While the predicted big bang yields of the other light elements were also calculated in the 1960 's, they were not considered important at that time, since it was assumed in the 1960's that these nuclei were made in more significant amounts in stars. ${ }^{18}$ However, work by our group at $\mathrm{Chicago}^{8}$, and others, thoroughly established big bang nucleosynthesis and turned it into a tool for probing the Universe, by showing that other light element abundances had 
major contributions from the big bang and that the effects of any stellar contributions could be removed by appropriate techniques. Today the big bang predictions for all four light isotopes are used to test the model and use it as a probe of conditions at early times.

In particular, it was demonstrated in the early 1970's that contrary to the ideas of the 1960 's, deuterium could not be made in any significant amount by any realistic contemporary astrophysical process ${ }^{19}$. The big bang deuterium yield decreases rapidly with increasing $\rho_{b}$. At high densities deuterium gets more completely converted to ${ }^{4} \mathrm{He}$; quantitatively this means that the present density of baryons must be below $\sim 5 \times 10^{-31} \mathrm{~g} / \mathrm{cm}^{3}$ in order for the big bang to have produced enough deuterium to explain the observed abundance. Similar, though more complex, arguments ${ }^{20}$ were also developed for ${ }^{3} \mathrm{He}$, and most recently for ' $\mathrm{Li}$, so that it can be said that only if the baryon density is between $2 \times 10^{-31}$ $\mathrm{g} / \mathrm{cm}^{3}$ and $5 \times 10^{-31} \mathrm{~g} / \mathrm{cm}^{3}$ are all the observed light element abundances consistent with the big bang yields. If the baryon density were outside of this narrow range, a significant disagreement between the big bang predictions and the observed abundances would result. To put this in perspective, it should be noted that for this range in densities, the predicted abundances for the four separate species cover a range from $25 \%$ to one part in $\sim 10^{10}$. The big bang yields all agree with only one freely adjustable parameter, $\rho_{b}$.

Recently, several non-standard scenarios of primordial nucleosynthesis have been proposed ${ }^{23}$; however, these scenarios with their additional adjustable parameters seem to be unable to account for the abundances of the 4 light isotopes, especially ${ }^{7} \mathrm{Li}$. This speaks to the remarkable success of the standard scenario of big bang nucleosynthesis.

This narrow range in baryon density for which concordance occurs is very interesting. Let us convert it into units of the critical cosmological density for the allowed range of Hubble expansion rates. From big bang nucleosynthesis ${ }^{19,20}$, it follows that the baryon density $\Omega_{B}$ is less than 0.12 and greater than 0.03 (once one includes ${ }^{21}$ age constraints on a flat Universe); that is the Universe cannot be closed with baryonic matter. If the Universe 
is truly at critical density, then non-baryonic matter is required. This argument has led to one of the major areas of research at the particle-cosmology interface, namely, the search for non-baryonic dark matter.

Another important conclusion regarding the allowed range in baryon density is that it is in very good agreement with the density implied from the dynamics of galaxies, including their dark halos. An early version of this argument, using only deuterium, was described over 15 years agn ${ }^{22}$. As time has gone on, the argument has strengthened and the fact remains that galactic dynamics and nucleosynthesis both suggest densities of about $10 \%$ of the critical density. Thus, if the Universe is indeed at critical density; as many believe, it requires that the bulk of the matter not be associated with galaxies and their halos, as well as being non-baryonic.

With the growing success of big bang nucleosynthesis, the predictions came under more scrutiny. In particular, the ${ }^{4} \mathrm{He}$ yield was examined in detail since it is the most abundant of the nuclei, and thus in principle it is the one which observers should be able to measure to highest accuracy. In addition, it is very sensitive to the $n / p$ ratio.

In the standard calculation it is assumed that photons, electrons, and the three known neutrino species (and their antiparticles) are present in the Universe at the time of nucleosynthesis. However, by doing the calculation with additional species of neutrinos we can see when ${ }^{4} \mathrm{He}$ yields exceed observational limits. The bound on ${ }^{4} \mathrm{He}$ comes from observations of helium in many different objects in the Universe. However, since ${ }^{4} \mathrm{He}$ is not only produced in the big bang but in stars as well, it is important to estimate what part of the helium in some astronomical object is primordial, from the big bang, and what part is due to stellar production after the big bang. To do this we $\mathrm{e}^{24}$ have found that the carbon content of the object is well suited for tracking the additional helium produced. Carbon is made in the same mass stars that also produce ${ }^{4} \mathrm{He}$, thus as the carbon abundance increases, so must the helium. (Other heavy elements such. as oxygen have been used previously, but these elements are not produced in the same mass stars as those that produce the bulk of 
the helium.) The extrapolation of helium to zero carbon content in an object should be a good estimate of the primordial helium. We obtain $\sim 0.235$ as our best estimate for the mass fraction of helium produced in the big bang. The upper bound is what is important here. We formally estimate a three standard deviation bound as 0.247 . In particular, it seems clear that the primordial ${ }^{4} \mathrm{He}$ was at least a little less than $25 \%$. Since objects have heary elements and possibly some associated extra-stellar produced helium and still have helium abundances of $25 \%$, this certainly seems like a very safe upper bound. In fact, if anything our estimates are on the high side due to possible srstematic errors, e.g., Page ${ }^{25}$ finds collisional excitation reduces the 0.235 to 0.233 .

We find (see Figure 6) that three (or two) types of neutrinos fit the data well, and a fourth is only marginally allowed if helium slightly exceeds the 3- $\sigma$ upper bound; any more neutrinos are strictly prohibited. Since each family contains a neutrino, we are saring that the total number of families is three or at most four. Thus, all the fundamental families of elementary particles may have been already discovered. Of course, this assumes that the neutrinos are "light," i.e., less massive than $\sim \mathrm{MeV}$.

\section{Supernova 198TA and Neutrino Counting}

Let us now compare this bound with the supernova constraint. As is now well appreciated, neutrinos were detected ${ }^{26}$ from SN 1987A by both Famiokande ${ }^{26}$ and IMB $^{27}$. Both of these $\mathrm{H}_{2} \mathrm{O}$ detectors are most sensitive to $\bar{\nu}_{e}+p \rightarrow n+\epsilon^{+}$because of its larger cross section.

If the $\bar{\nu}_{e}$ flux is assumed to come from a Fermi-Dirac (F-D) distribution at temperature $T$ and total $\bar{\nu}_{e}$ energy, $\epsilon_{\bar{\nu} e}$, both IMB and Kamiokande are simultaneously fit with $T \sim 4$ to $4.5 \mathrm{MeV}$ and $\epsilon_{\bar{\nu}_{e}} \sim 3$ to $4.5 \times 10^{52}$ ergs. These figures are in remarkable agreement with the standard model ${ }^{28}$ for gravitational core collapse of a massive star, if $N_{\nu}=3$. Thus, we have confidence that we have witnessed such a core collapse, and that we have a good understanding of its physics. Let us now turn the argument around and see how sensitive 
our expected fluxes are to $N_{\nu}$.

In a collapse to a neutron star, the binding energy, $\epsilon_{B}$, must be radiated as neutrinos. The initial neutronization burst of $\nu_{e}$ 's carries away a fraction $f_{n} \lesssim 10 \%$ of $\epsilon_{B}$ on a timescale of $\lesssim 10 \mathrm{~ms}$. The remaining energy comes out in thermal $\nu \bar{\nu}$ pairs from reactions like

$$
c^{+} e^{-} \rightarrow \nu \bar{\nu}
$$

where through neutral currents all species of neutrinos with $m_{\nu} \lesssim 10 \mathrm{MeV}$ are emitted.

Since electron scattering rates are small compared to $\bar{v}_{\epsilon}$ capture, even with five times more free electrons than protons, at most we expect one or two scattering events in the detectors for a SN at $50 \mathrm{Kpc}$ (distance to LMC). Thus, the detectable fraction of $\epsilon_{B}$ is $\epsilon_{\bar{\nu}_{e}}$ where

$$
\epsilon_{\bar{\nu}_{e}} \approx \frac{\left(1-f_{n}\right)}{2 N_{\nu}} \epsilon_{B}
$$

assuming an equipartition of energy emitted in the various neutrino species, as is found in the detailed models. (While average energy per neutrino is higher for $\nu_{\mu}$ and $\nu_{\tau}$, their flux is correspondingly lower.) The number of counts, $n$, one expects in a detector of mass, $M_{D}$, is

$$
n \approx \frac{\epsilon_{\bar{\nu}_{e}}}{\left\langle E_{\bar{\nu}_{e}}\right.} \frac{\langle\sigma\rangle}{4 \pi R^{2}} \frac{2 M_{D}}{18 m_{p}}
$$

where $m_{p}$ is the proton mass, $R \approx 50 \mathrm{Kpc}$ is the distance to LMC, $\left\langle E_{\bar{\nu}_{e}}\right\rangle$ is the average $\bar{\nu}_{e}$ energy, and $\langle\sigma\rangle$ is the cross section appropriately averaged over a F-D distribution with appropriate threshold factors and efficiencies taken into account. The temperat ure of $\nu_{e}$ 's is found to be $\sim 3.2 \mathrm{MeV}\left(\left\langle E_{\nu}\right\rangle \approx 10 \mathrm{MeV}\right)$ to good accuracy. Temperatures are very insensitive to model parameters being determined by microphysics at the neutrinosphere ${ }^{14}$. The temperature for $\bar{\nu}_{e}$ 's is somewhat higher due to the smaller opacities at late times as protons disappear in the core, thereby minimizing charged current interactions. This enables the $\bar{\nu}_{e}$ 's to come from deeper in the star. Mayle et al. ${ }^{28}$ find $T_{\bar{\nu}_{\varepsilon}} \sim 4 \mathrm{MeV}$ in good agreement with the temperature inferred from the observations. (They do find a higher than thermal high energy tail to the distribution which can effect the high threshold IMB 
but not Kamioka.) For detectors like Kamiokande where the threshold is well below the peak of the cross section weighted distribution, it is reasonable to use

$$
\langle\sigma\rangle \approx \sigma_{0}\left(g_{v}^{2}+3 g_{A}^{2}\right) 12 T_{\bar{\nu}_{e}}^{2}
$$

(For IMB a more careful procedure must be applied due to its high threshold.) Substituting into $\mathrm{Eq}(56)$ yields

$$
n=\frac{5.2}{\left(N_{\nu} / 3\right)}\left(\frac{1-f_{n}}{2 \times 10^{53} \mathrm{ergs}}\right)\left(\frac{T_{\bar{\nu}_{e}}}{4 \mathrm{MeV}}\right)\left(\frac{M_{D}}{\text { kions }}\right)\left(\frac{50 \mathrm{Kpc}}{R}\right)^{2}
$$

which for $M_{D}=2.14$ ktons (Kamiolia) we obtain a prediction of 11 counts for $N_{\nu}=3$. While they actually observe 11, one should weigh their counts by efficiency effects to obtain $16.5 \pm 5$. Solving for $N_{\nu}$ yields

$$
N_{\nu}=(2 \pm 0.6)\left[\left(\frac{T_{\bar{\nu}_{e}}}{4 \mathrm{MeV}}\right)\left(\frac{\epsilon_{B}}{2 \times 10^{53} \mathrm{ergs}}\right)\left(\frac{1-f_{n}}{0.9}\right)\left(\frac{50 \mathrm{Kpc}}{R}\right)\right]
$$

Let us now see how high we can push this. While models can be found with $f_{n}>0.1$, it is obvious that $1-f_{n}$ can never exceed unity. The effective $T_{\bar{l}^{\prime} e}$, as used abore, varies by $\lesssim 25 \%$. The binding energy for $1.4 M_{\odot}$ neutron stars (the mass of the collapsing core) is found to vary from 1.5 to $3 \times 10^{53} \mathrm{ergs}$ for a wide range of equation-of-state 29 . Thus, we choose $3 \times 10^{53} \mathrm{ergs}\left(4 \times 10^{53} \mathrm{ergs}\right)$ as an (extreme) upper bound. The distance to the LMC varies in the astronomical literature by $<7 \%$. We'll adopt an extreme limit of $10 \%$ consistent with current SN 1987 A determination of the distance ${ }^{30}$. Combining all these extreme value yields

$$
N_{\nu}<6.6(8.9)
$$

A more careful calculation taking into account different thresholds for both IMB and Kamiokande to obtain measured $\epsilon_{\bar{\nu}_{e}}$ for predicted yields at the $T_{\bar{\nu}_{e}}$ inferred from the data yields essentially the same result $\left(N_{\nu} \lesssim 6.7(9.0)\right)$ as given above. Thus, SN 19STA gives a limit to $N_{\nu}$ comparable to accelerator experiments but not as strong as the big bang nucleosynthesis limits. 


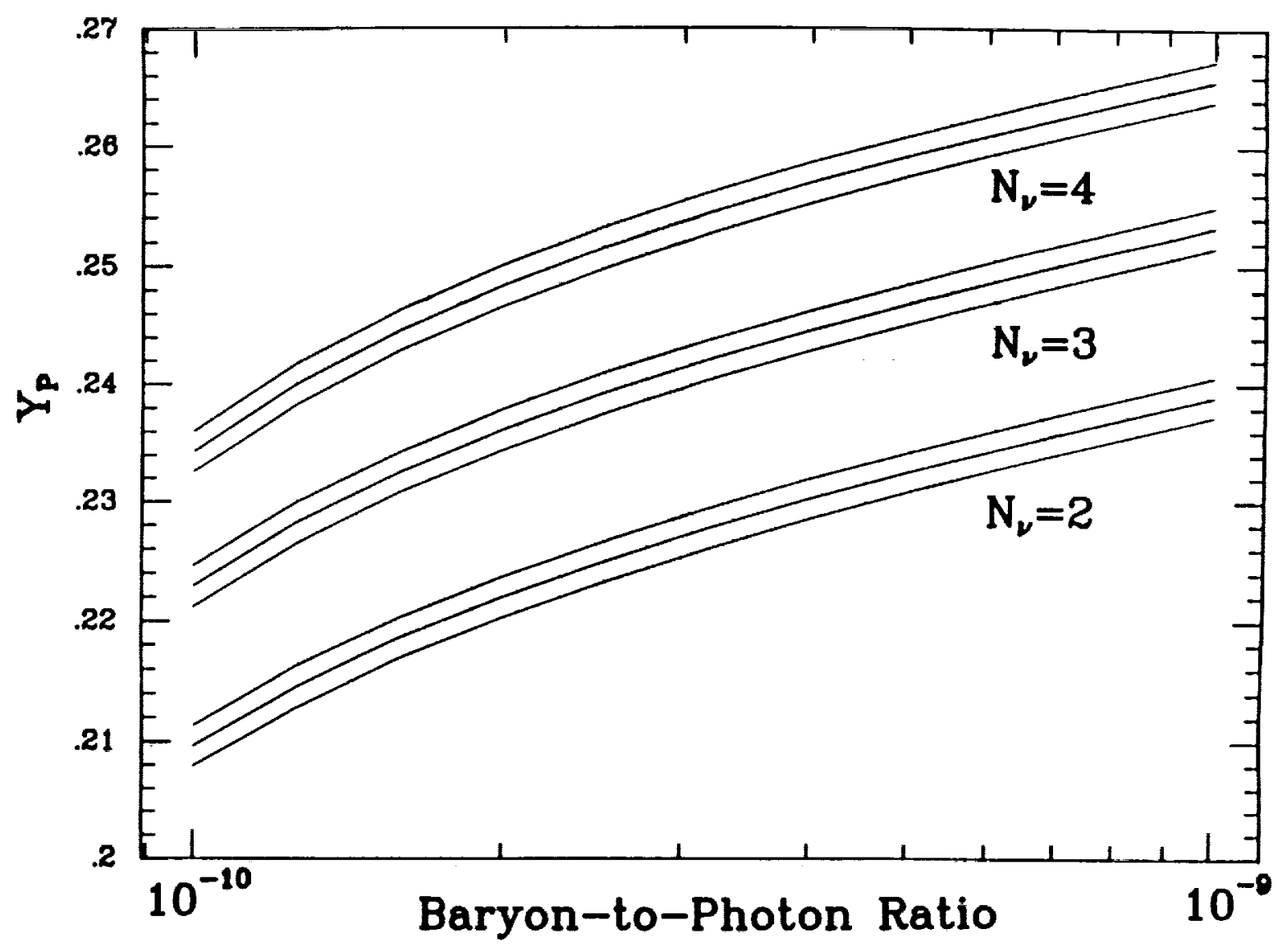

Fig. 6: Helium mass fraction versus the baryon-to-photon ratio $\eta$. The lower bound of $2 \times 10^{-10}$ derives from the ${ }^{3} \mathrm{He}+D$ and ${ }^{7} \mathrm{Li}$ constraints, and the upper bound of $7 \times 10^{-10}$ from the $D$ and ${ }^{7} L i$ constraints. The three lines for each neutrino family correspond to neutron half-lives of 10.4, 10.5, and 10.6 minutes (from ref. 8). 


\section{Other Constraints from SN1987A}

SN1987A has proven to be an amazing neutrino laboratory. In addition to the previously mentioned limits, it has placed limits to the charge and magnetic moment of the neutrino that exceed current laboratory limits and its constraint to the mass of $\nu_{\epsilon}$ is comparable to the best laboratory limits. Let us briefly review these bounds.

\section{Magnetic Moment}

Barbieri and Mohapatra, and Lattimer and Cooperstein ${ }^{31}$ have shown that the observation of $\bar{\nu}_{e}$ 's from SN 198TA constrains the value of the magnetic moment of the neutrino to $\lesssim 10^{-11} \mu_{B}$. The argument is twofold, involving in a crucial way the fact the interaction cross section of right-handed neutrinos must be significantly weaker than those of lefthanded neutrinos. (Right-handed Dirac neutrinos must interact more weakly so that they do not get counted in the big bang nucleosynthesis argument ${ }^{32}$.) First, there is the limit from cooling the proto-neutron star too rapidly if $\nu_{L}$ 's can change to $\nu_{R}$ s $\mathrm{s}$ as a result of magnetic moment interactions in the proto-neutron star core. Second, there is the effect that $\mathbf{a}$ flipped $\nu_{R}$ can escape from the higher temperature inner core and then get flipped back to a $\nu_{L}$ by the intergalactic magnetic field. This latter situation could yield $70 \mathrm{MeV}$ $\bar{\nu}_{e}$ 's which were definitely not detected. It is argued that these processes limit the magnetic moment to $\lesssim 10^{-13}$ with $10^{-11} \mu_{B}$ as an extreme upper limit. However, Okun ${ }^{33}$ has argued that these arguments can be circumvented if the magnetic moment is not static but is a majoron transition moment or if an appropriate MSW mixing ${ }^{36}$ of neutrino species also occurs in the supernova.

\section{Neutrino Charge}

Barbelini and Cocconi ${ }^{34}$ have argued that the absolute value of any neutrino charge must be $\lesssim 10^{-17}|e|$; otherwise electrostatic repulsion would have spread the neutrino burst greater than $\sim 10 \mathrm{sec}$ on its 170,000 yr. flight from the LMC to earth. 


\section{Neutrino Mass}

Since the observed neutrino burst was relatively narrow ( $\lesssim 10 \mathrm{sec}$ ), despite energies which spanned a range of about a factor of two, it is obvious that any neutrino rest mass must be very small. While the relationship between mass, timespread and energy is a simple one, the key here is to decide on the significance of the time and energy spread, and to estimate what the intrinsic spread was in the neutrino burst in the absence of finite masses.

The crucial, but simple, relationship at the heart of any analysis to constrain the $\nu_{\epsilon}$ mass from the IMB and Kamiokande data is that for the time delay suffered by a neutrino during its flight to earth:

$$
\Delta t \cong \frac{1}{2} \frac{R}{c} \frac{m^{2}}{E^{2}} \simeq 2.6 \sec \frac{(m / 10 \mathrm{eV})^{2}}{(E / 10 \mathrm{MeV})^{2}}
$$

From this simple equation for $\Delta t$, it is clear that any mass constraint which follows will be in the general range of about $20 \mathrm{eV}$, or so, which is comparable to existing laboratory limits. Given the sparseness of the data set (19 events in total), the subtleties of the detectors (response, thresholds, etc.) and the absence of a very specific, well-accepted standard model of the initial cooling, it is not surprising that many authors have 'derived' limits (and even values!) for the $\nu_{\epsilon}$ mass ranging from a few $\mathrm{eV}$ to $30 \mathrm{eV}$. The most extensive and careful analyses to date ${ }^{35}$ provide limits of around $20 \mathrm{eV}-25 \mathrm{eV}$. While SN $1987 \mathrm{~A}$ has not really improved existing bounds, it is interesting that the constraint which is found is comparable to the present laboratory limits.

\section{Neutrino Mixing}

Neutrino mixing has been proposed as a solution to the solar neutrino problem ${ }^{36}$ and the Homestake and Kamiokande observations of solar neutrinos place constraints on allowed mixing parameters ${ }^{37}$. A supernova could potentially also test neutrino mixing ${ }^{38}$. If neutrino mixing occurs between supernova emission and detection, it can obriously alter 
the detected neutrino signal.

If MSW mixing is indeed the solution to the solar neutrino problem, then only $\nu_{\epsilon} \leftrightarrow$ $\nu_{\mu}\left(\nu_{\tau}\right)$ mixing is possible and not $\bar{\nu}_{\epsilon} \rightarrow \bar{\nu}_{\mu}\left(\bar{\nu}_{\tau}\right)$. Thus, the solar neutrino solution would not affect the $\bar{\nu}_{e}$ flux. However, it could deplete the initial neutronization burst. Unfortunately, there is no conclusive evidence that even a single $\nu_{e}+\epsilon^{-} \rightarrow \nu_{\epsilon}+\epsilon^{-}$scattering event associated with the neutronization burst was seen.

If we drop the solar neutrino solution and go to general MSW mixing, then we can mix $\bar{\nu}_{\mu}\left(\bar{\nu}_{\tau}\right)$ into $\bar{\nu}_{\epsilon}$, which might enhance the energy slightly; but would otherwise do little. No effect would occur for the electron scattering $\nu_{\varepsilon}$ 's. Thus no definitive statement can be made from SN 198TA about neutrino mixing and oscillations.

\section{Secret Interactions}

Precious little is known about any interactions neutrinos may have beyond the standard weak interactions, e.g., additional neutrino-neutrino interactions as in the Majoron model. Since neutrinos from the supernora traversed 170,000 light years through the cosmic seas of relic neutrinos (and perhaps other particles such as majorons) without apparent attenuation, any unknown (i.e., secret) interactions they might have with neutrinos (or other particles in the sea of relics) can be constrained:

$$
\sigma_{\text {secret }} \lesssim 10^{-25} \mathrm{~cm}^{2}
$$

\section{Radiative Decays}

The fluence of neutrinos from SN1987A was enormous, $\sim 10^{10} \mathrm{~cm}^{-2}$ per species (integrated over the observed burst). On the other hand there was no observation (above instrument background) of any high energy $\gamma$ rays: based upon the data of the Gamma Ray Spectrometer aboard the Solar Maximum Mission and $\gamma$ ray detectors on the Pioneer Venus Orbiter a $\gamma$-ray fluence limit for the same time period of $\lesssim 1 \mathrm{~cm}^{-2}$ follows. 
This means that less than about 1 in $10^{10}$ of the supernova neutrinos could have decayed producing a $\gamma$-ray. From these non-observations of $\gamma$-rays a limit of

$$
\tau_{s e c} / m_{\epsilon} V^{\prime} \gtrsim 2 \times 10^{15} B_{\gamma}
$$

can be set to the radiative decay of any neutrino species. Here $B_{\gamma}$ is the branching ratio for the radiative decay mode.

\section{Acknowledgments}

This work was supported in part by NSF, NASA, and DOE at The University of Chicago and by the NASA/Fermilab Astrophysics Center (NAGW-1340).

\section{References}

1. E. Kolb and M. Turner, The Early Universe (Addison Wesley, Redwood City, 1989).

2. R. Cowsik and J. McClelland, Phys. Rev. Lett. 29,669 (1972). It was first derived by G. Gerstein and Ya. B. Zel'dovich, Zh. Eksp. Teor. Fiz. Pis'ma Red. 4, 174 (1966); and it was also discussed by G. Marx and A. Szalay, in Neutrino '72, eds. A. Frenkel and G. Marx (OMKDT-Technoinform, Budapest, 1972), p. 123.

3. B. W. Lee and S. Weinberg, Phys. Rev. Lett. 39, 165 (1977), although it was discovered independently by many people: P. Hut, Phys. Lett. 69B, 85 (1977); K. Sato and H. Kobayashi, Prog. Theor. Phys. 58, 1775 (1977); M. I. Vysotskii, A. D. Dolgov, and Ya. B. Zel'dorich, JETP Lett. 26, 188 (1977) and the basic argument was first noted by Ya. B. Zel'dovich, Adv. Astron. Astrophys. 3, 241 (1965); and H. Y. Chiu, Phys. Rev. Lett. 17, 712 (1960). 
4. J. Silk, K. Olive and M. Srednicki, Phys. Rev. Lett. 55, 257 (1985); T. Gaisser, G. Steigman and S. Tilav, Phys. Rev. D34, 2206 (1986); K. Ng. K. Olive and M. Srednicki, Phys. Lett. 188B, 138 (1987).

5. M. I. Vysotsky, Ya. B. Zel'dovich, M. Yu. Khlopov, and V. M. Chechetkin, Zh. Eksp. Teor. Fiz. Pis'ma 26, 200 (1977); 27, 533 (1978); K. Sato and H. Kobayashi, Prog. Theor. Phys. 58, 1775 (1977); D. A. Dicus, E. W. Kolb, and V. L. Teplitz, Phys. Rev. Lett. 39, 168; (1977) Ap. J. 221, 327 (1978); T. Goldman and G. J. Stephenson, Phys. Rev. D 16, 2256 (197T).

6. K. Freese, E. W. Kolb, and M. S. Turner, Phys. Rev. D 27, 1689 (1983); G. Steigman and M. S. Turner, Nucl. Phys. 253B, 375 (1985).

7. K. Sato and H. Tobayashi, Prog. Theor. Phys. 58, 1775 (1977); J. E. Gunn, B. W. Lee, I. Lerche, D. X. Schramm, and G. Steigman. Ap. J. 223, 1015 (1978): D. A. Dicus, E. W. Kolb, and V. L. Teplitz, Ap. J. 221, 32T (1978); R. Cowsik, Phys. Rev. Lett. 39, 784 (197亍).

8. J. Yang, M. Turner, G. Steigman, D. Schramm, and K. Olive Ap. J. 281, 493 (1984), and references therein.

9. D. Lindley, MNRAS, 188, 15 (1979).

10. K. Sato and H. Kobayashi, Prog. Theor. Phys. 58, 1775 (1977); D. A. Dicus, E. W. Kolb, V. L. Teplitz, and R. V. Wagoner, Phys. Rev. D 17, 1529 (1978); S. Miyama and K. Sato, Prog. Theor. Phys. 60,1703 (1977).

11. R. Cowsik, Phys. Rev. Lett. 39, 784 (1977); S. W. Falk and D. N. Schramm, Phys. Lett. 79B, 511 (1978); M. A. B. Beg, W. J. Marciano, and M. Ruderman, Phys. Rev. D 17, 1395 (1978); M. Fukugita and S. Yazaki, Phys. Rev. D 36. 3817 (1987). The type II supernova rate is discussed in G. A. Tammann, Ann. NY Acad. Sci. 302, 61 (1977) and S, van den Bergh, R. D. McClure, and R. Evans, Ap. J. 323, 44 (1987). 
The limits based upon observations of SN198TA are discussed by E. W. Kolb and M. S. Turner Phys. Rev. Lett. 62, 509 (1989); R. Cowsik, P. Hoflich, and D. N. Schramm, Phys. Lett B., in press (1990).

12. D. Cline, D. Schramm, and G. Steigman, Comments Nuc. B Part. Physics A 17, $145(1986)$.

13. G. Steigman, D. Schramm, and J. Gunn, Phys. Lett. B66, 202 (1977); G. Steigman, K. Olive, M. Turner, and D. Schramm, Phys. Lett. 175B, 33 (1986). Also see the earlier work by V. Schvartzman (1969) JETP Lett 9, 184; P. J. E. Peebles, Phys. Rev. Lett. 16, 410 (1966); F. Hoyle and R. Tayler, Nature 203, 1108 (1964).

14. D. Schramm, Comments Nuc. \& Part. Physics A 17, 239 (19Si).

15. F. Hoyle and R.Tayler, Nature 203, 1108 (1964).

16. P. J. E. Peebles, Phys. Rev. Lett. 16. 410 (1960).

17. R. Wagoner, W. Fowler, and F. Hoyle, Ap. J. 148, 3 (1967).

18. W. Fowler, J. Greenstein, and F. Hoyle, Geophys. J. R.A.S. 6, 148 (1962).

19. R. Epstein, J. Lattimer, and D. Schramm, Nature 263, 198 (19i6).

20. L. Kawano, D. Schramm, and G. Steigman, Ap. J. 327, 750 (1987), and references therein.

21. K. Freese and D. Schramm, Nucl. Phys. B233, 16 (1984).

22. J. Gott, J. Gunn, D. Schramm, and B. Tinsley, Ap. J. 194, 543 (1974).

23. R. Scherrer, J. Applegate, and C. Hogan, Phys. Rev. D.35, 1151 (1987); C. Alcock, G. Fuller, and G. Mathews, Ap. J. 320, 439 (19Si); S. Dimopoulos, R. Esmailzadeh, L.Hall, and G. Starkman, Phy. Rev. Lett 60, 7 (1988). 
24. J. Gallagher, G. Steigman, and D. Schramm, Comments on Astron. \& Astrophys. in press (1989).

25. B. Pagel, Proc. 1987 Recontre de Moriond, Astrophysics, ed. J. Audouze (1987).

26. K. Hirata, et al., Phys. Rev. Lett. 58, 1490 (1987).

27. R. Bionta, et al., Phys. Rev. Lett. 58, 1494 (1987).

28. R. Mayle, J. Wilson, and D. Schramm, Ap. J. 318, 288 (1987); D. Schramm, J. Wilson, and R. Mayle, in Proc. 1st International Symposium on Underground Physics (1985).

29. W. Arnett and R. Bowers, Ap. J. 33415 (1977).

30. R. Wagoner, in Proc. Berkeley Workshop on Particle/Astrophysics, ed. E. Norman, World Scientific, in press (1988).

31. R. Barbieri and R. N. Mohapatra, Phys. Rev. Lett. 61, 27 (1988); J. Lattimer and J. Cooperstein, Phys. Rev. Lett. 61, 23 (1988).

32. K. Olive, D. Schramm, and G. Steigman, Nuc. Phys. 180, 497 (1981).

33. L. Okun, Proc. Neutrino 88 Cambridge, Mass., in press (1988).

34. C. Barbelini and G. Cocconni, Europhys. Lett., in press (1988).

35. D. Lamb, T. Loredo, and F. Melia, Phys. Rev. D., in press (1989).

36. S. P. Mikheyev and A. Yu Smirnov, $n$ Nuovo Cimento 96, 17 (1986); L. Wolfenstein, Phys Rev D 17, 2364 (1978).

37. E. Kolb and M. Turner, Phys. Rev. Lett D36, 2895 (198i).

38. T. Walker and D. Schramm, Phys. Lett. B. 195B, 331 (1987). 
